Supporting Information

\title{
Accessing In Situ Photocorrosion under Realistic Light Conditions: Photoelectrochemical Scanning Flow Cell Coupled to Online ICP-MS
}

\author{
Ken J. Jenewein, ${ }^{\text {a,b, }, *}$ Attila Kormányos, ${ }^{a}$ Julius Knöppel, ${ }^{\mathrm{a}, \mathrm{b}}$ Karl J. J. Mayrhofer, ${ }^{\mathrm{a}, \mathrm{b}}$ and Serhiy \\ Cherevko $^{\mathrm{a}, *}$ \\ ${ }^{a}$ Forschungszentrum Jülich GmbH, Helmholtz Institute Erlangen-Nürnberg for Renewable Energy (IEK- \\ 11), Egerlandstrasse 3, 91058 Erlangen, Germany \\ ${ }^{b}$ Department of Chemical and Biological Engineering, Friedrich-Alexander-Universität Erlangen- \\ Nürnberg, Egerlandstrasse 3, 91058 Erlangen, Germany \\ *corresponding authors k.jenewein@fz-juelich.de
}

s.cherevko@fz-juelich.de

\section{Flow profile simulations using COMSOL Multiphysics}

All simulations were performed using Finite Element software COMSOL Multiphysics 5.5. The simplified SFC design was constructed in a 3D geometry. Flow channel diameters were $2.2 \mathrm{~mm}$ with an inlet diameter of $1.02 \mathrm{~mm}$ to match the inner diameter of a Tygon tubing used in the present PEC-ICP-MS setup. The element size for the mesh was set to "Finer" and was physics-controlled. Water was used as the fluid material and was introduced with an inlet flow velocity of $0.428 \mathrm{~cm} \mathrm{~s}^{-1}$ to mimic employed flow rates. The velocity on the edges was constrained to zero to match the no-slip conditions on the walls. The outlet pressure was set to be close to atmospheric $\left(10^{5} \mathrm{~Pa}\right)$. The electrolyte flow profile was calculated using incompressible Navier-Stokes equations with a laminar flow interface since Reynolds numbers were assumed to be low enough based on previous reports. ${ }^{1}$ The temperature was kept at $293.15 \mathrm{~K}$.

\section{Sample preparation}

The preparation of spray-coated $\mathrm{WO}_{3}$ was described in a previous study. ${ }^{2}$ In short, $5 \mathrm{~g}$ of tungsten powder (fine powder $99+\%$, Merck) was dissolved in $25 \mathrm{~mL}$ of $\mathrm{H}_{2} \mathrm{O}_{2}(30 \%$, Merck). Upon complete dissolution, the solution was diluted with Milli-Q water and heated under continuous stirring. After the solution evaporated down to $20 \mathrm{~mL}$, isopropyl alcohol was added to dilute to $200 \mathrm{~mL}$. A spray coater (ExactaCoat, SonoTek) equipped with a spray nozzle (AccuMist, Sonotek) was used to apply the $\mathrm{WO}_{3}$ layers on glass slides coated with fluorine-doped tin oxide. The hot plate temperature was adjusted to $80^{\circ} \mathrm{C}$, and the ink was sprayed with a flow rate of $0.33 \mathrm{~mL} \mathrm{~min}^{-1}$ at an ultrasonication power of $5 \mathrm{~W}$, a nozzle height of $37 \mathrm{~mm}$, and a traverse speed of $140 \mathrm{~mm} \mathrm{~s}^{-1}$ in a meander-shaped pattern with $1.5 \mathrm{~mm}$ pitch size. The electrodes were spray-coated with 24 cycles. Afterwards, the samples were annealed at $500{ }^{\circ} \mathrm{C}$ for 1 hour with a heating ramp of $2^{\circ} \mathrm{C} \mathrm{min}$. 


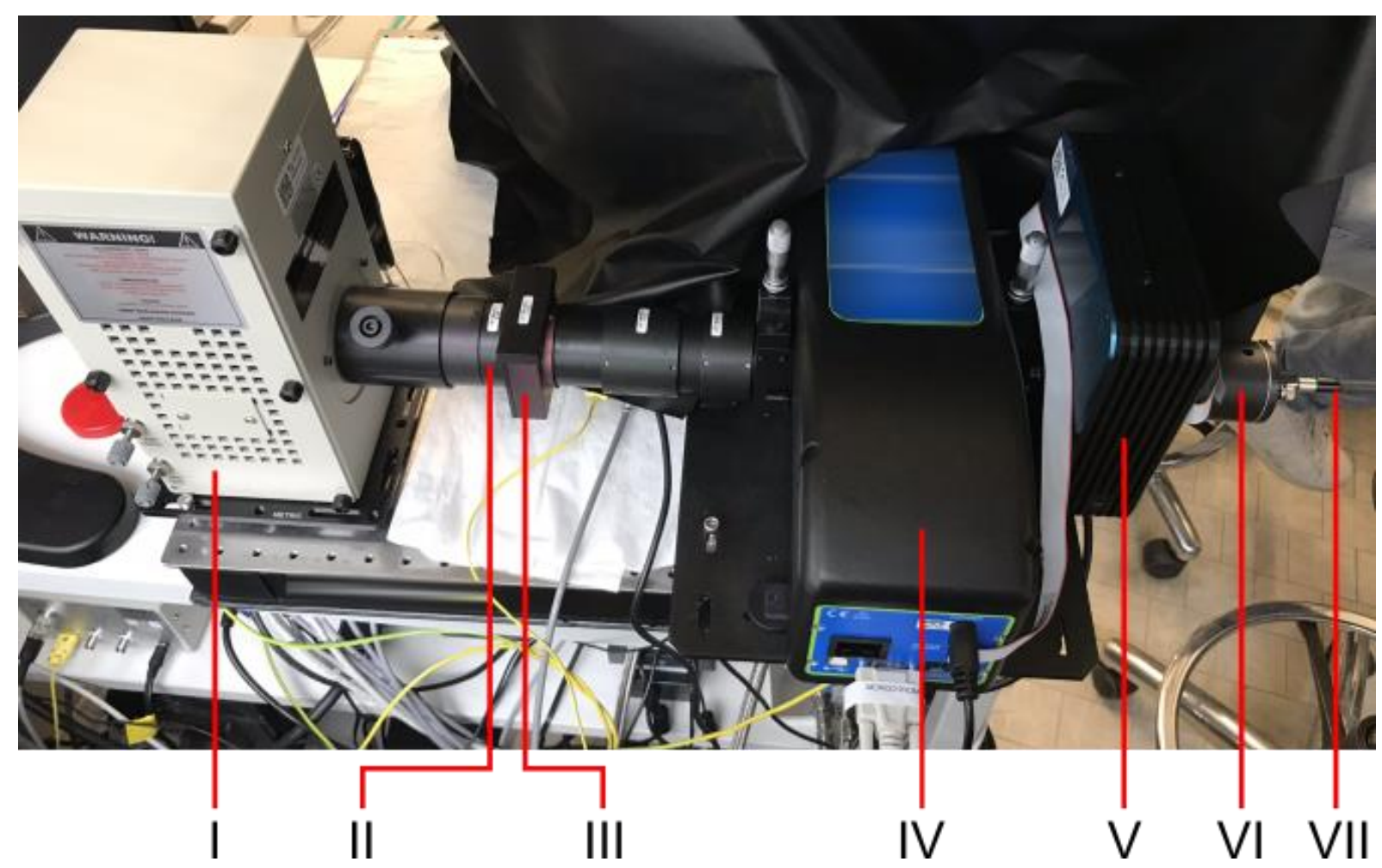

Figure S1 Photograph of light components used in the PEC-SFC setup. (I) $300 \mathrm{~W}$ ozone-free Xe lamp, (II) focusing lens, (III) AM 1.5 G filter, (IV) monochromator, (V) filter wheel with different cutoff filters, (VI) focusing assembly, and (VII) liquid light guide.

a

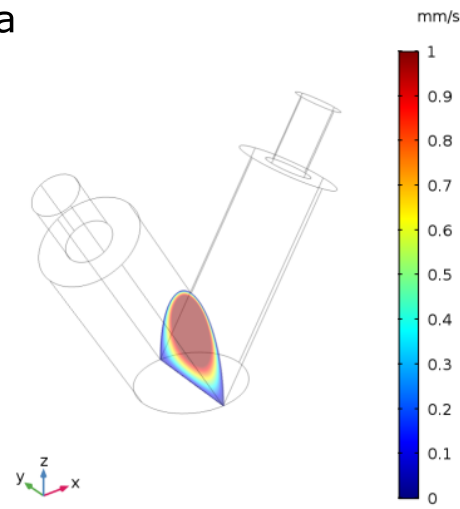

b

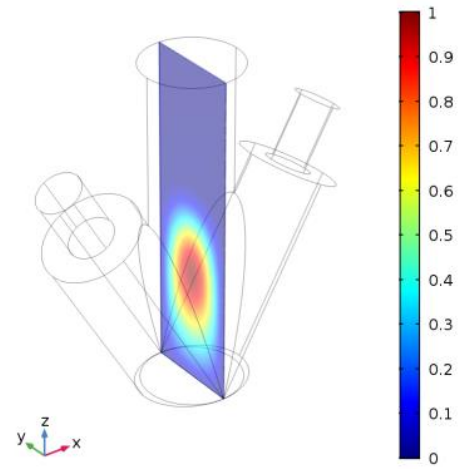

C

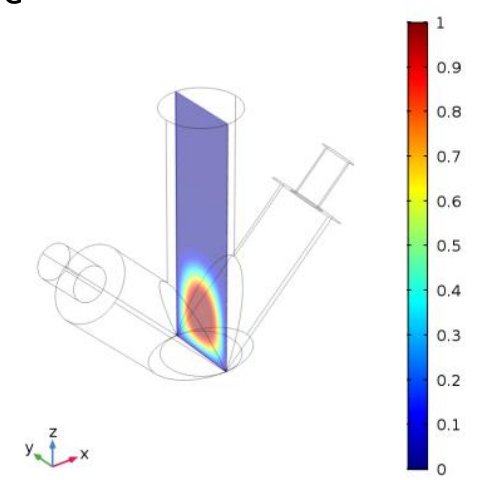

Figure S2 Flow velocity simulation for SFC design (a) without and (b, c) with light channel using flow channels angled at $(a, b)$ $60^{\circ}$ and $(\mathrm{c}) 90^{\circ}$. 
a

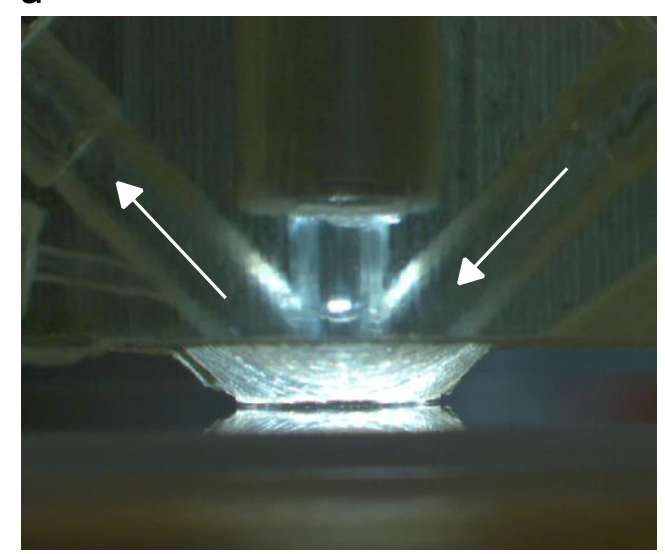

b

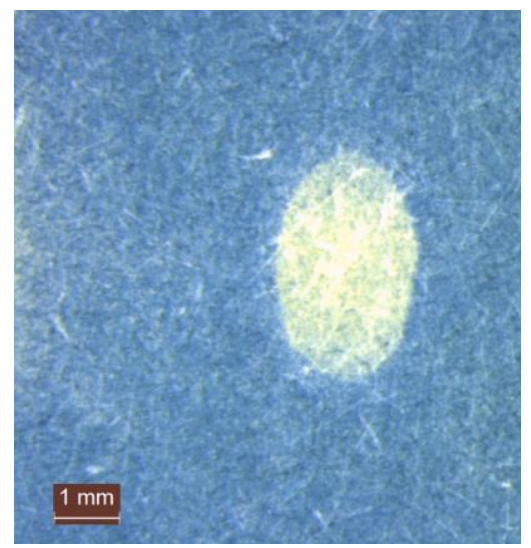

Figure S3 (a) Photograph of transparent 3D printed PEC-SFC with arrows indicating flow direction of $0.1 \mathrm{M} \mathrm{HCIO}_{4}$. (b) Photograph of illuminated area on a UV-sensitive paper by PEC-SFC
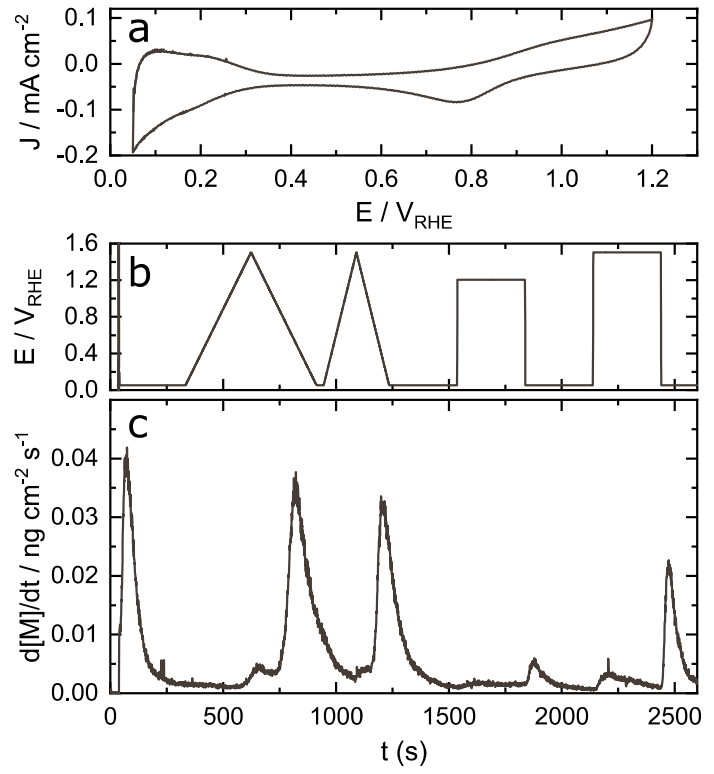

Figure S4 (a) Pt-Poly CVs with a scan rate of $10 \mathrm{mV} \mathrm{s}^{-1}$. Gas pocket of PEC-SFC was purged with Ar at $120 \mathrm{~mL} \mathrm{~min}^{-1}$. (b) Potential protocol for polycrystalline Pt dissolution with 5 and $10 \mathrm{mV} \mathrm{s}^{-1}$ for the first and second CV, respectively. Last two CA pulses held for five minutes with an upper potential limit of 1.2 and $1.5 \mathrm{~V}_{\mathrm{RHE}}$, respectively. (c) Corresponding dissolution of $\mathrm{Pt}$ recorded with a PEC-SFC in dark conditions. All measurements were conducted in Ar-saturated $0.1 \mathrm{M} \mathrm{HClO}_{4}$. 


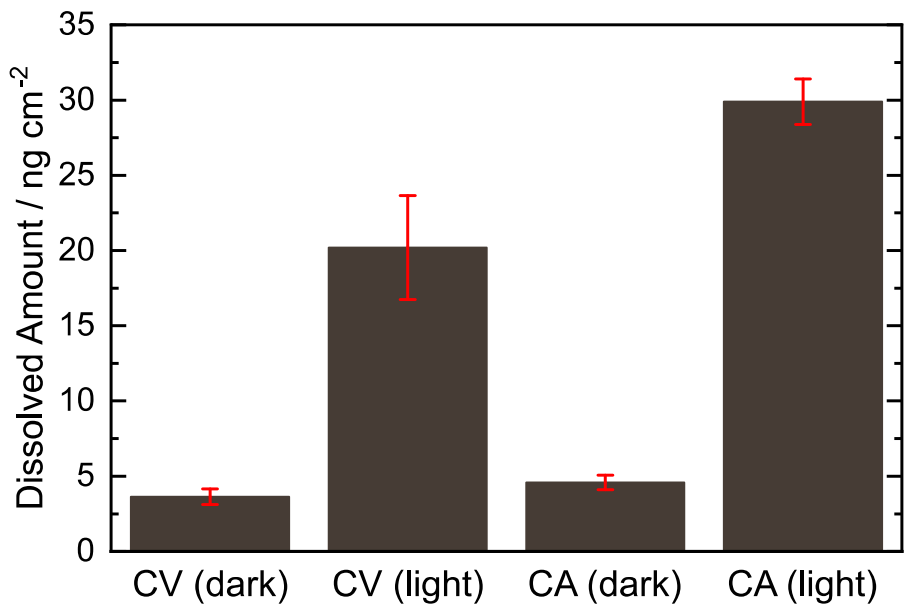

Figure S5 Reproducibility of measured W dissolution. Error bars were determined from at least three individual measurements performed on fresh $\mathrm{WO}_{3}$ spots each.

\section{References}

1. Shkirskiy, V.; Speck, F. D.; Kulyk, N.; Cherevko, S., On the Time Resolution of Electrochemical Scanning Flow Cell Coupled to Downstream Analysis. J. Electrochem. Soc. 2019, 166 (16), H866-H870.

2. Knöppel, J.; Kormányos, A.; Mayerhöfer, B.; Hofer, A.; Bierling, M.; Bachmann, J.; Thiele, S.; Cherevko, S., Photocorrosion of WO3 Photoanodes in Different Electrolytes. ACS Physical Chemistry Au 2021. 